\title{
Impact of obesity and Ala16Val MnSOD polymorphism interaction on lipid, inflammatory and oxidative blood biomarkers
}

\author{
Maria Fernanda Manica-Cattani ${ }^{1}$, Francine Carla Cadoná ${ }^{2}$, Raul de Oliveira ${ }^{1}$, Tális da Silva ${ }^{1}$, \\ Alencar Kolinski Machado ${ }^{3}$, Fernanda Barbisan ${ }^{3}$, Marta Maria Medeiros Frescura Duarte ${ }^{1}$, \\ Ivana Beatrice Mânica da Cruz ${ }^{1,2,3^{*}}$
}

\footnotetext{
${ }^{1}$ Biogenomic Laboratory, Center of Science of Health, Federal University of Santa Maria, Santa Maria, Brazil

${ }^{2}$ Post-Graduate Program in Toxicology Biochemistry, Federal University of Santa Maria, Santa Maria, Brazil

${ }^{3}$ Post-Graduate Program in Pharmacology, Federal University of Santa Maria, Santa Maria, Brazil

${ }^{4}$ Post-Graduate Program in Pathology, Federal University of Health Science of Porto Alegre, Porto Alegre, Brazil

Email: ${ }^{*}$ marymanica@yahoo.com.br
}

Received 25 September 2012; revised 24 October 2012; accepted 22 November 2012

\begin{abstract}
Previous investigations suggest association between obesity and Ala16Val MnSOD gene polymorphism. The $V$ allele produces enzyme which not catalyze the superoxide anion efficiently as occurs with $A$ allele. As obesity is related to development of other metabolic disorders we performed a study that analyzed the effect of interaction between Ala16Val MnSOD polymorphism and obesity on lipid, oxidative and inflammatory biomarkers of adult subjects. The study enrolled 161 volunteers as categorized in six groups with different genotypes: Obeses with different genotypes (AAO, VVO and AVO) and nonobese (AANO, VVNO and AVNO). In general the group AANO presented lower values whereas VVO presented higher values of biomarkers analyzed. These results suggest that oxidative metabolism influenced by genetic status could to minimize or maximize the obesity effects on lipid, oxidative and inflammatory biomarkers that are also implicated in the genesis of important dysfunctions and diseases as atherosclerosis, diabetes 2 and cardiovascular morbidities.
\end{abstract}

Keywords: Ala16Val MnSOD Polymorphism; Obesity; Oxidative Stress; Inflammation; Oxidized LDL;

Anti-Oxidized LDL; Hs-PCR

\section{INTRODUCTION}

Currently is accepted that moderate levels of reactive oxygen species (ROS) are important signalizing cell molecules involved for cellular proliferation and host defense. However, excessive amounts of ROS molecules

"Corresponding author. cause oxidative stress that is harmful to cellular metabolism and cause unwanted events, such as DNA, RNA, and proteins damage, and induction of apoptosis or necrosis [1]. Cellular alterations caused by oxidative stress have been related to genesis of several morbidities including the obesity [2].

The biological basis to ROS increasing by the cell metabolism involves, mainly the mitochondria. This organelle plays a central role in energy homeostasis by metabolizing nutrients producing ATP and heat, also produce ROS. During respiration, some electrons released from the mitochondrial electron transport chain incomepletely reduce $\mathrm{O}_{2}$ to superoxide anion. The superoxide is a ROS molecule and must be maintained at nanomolar concentrations to avoid cellular damages [3].

The endogenous control of cellular superoxide levels is made by superoxide dismutase (SOD) enzymes. SODs have the capacity to dismutate the superoxide to hydrogen peroxide $\left(\mathrm{H}_{2} \mathrm{O}_{2}\right)$ and this molecule is further catalyzed in $\mathrm{H}_{2} \mathrm{O}$ and $\mathrm{O}_{2}$ by enzymes such as catalase (CAT) and glutathione peroxidases (GPX). The SOD enzymes family is comprised of MnSOD (SOD2) located in the mitochondrial matrix, and $\mathrm{Cu} / \mathrm{ZnSOD}$ located in the mitochondrial inter membrane space, cytosol and extracellular space (SOD1 and SOD3) [4].

Obesity is closely associated with an increased risk for metabolic and cardiovascular diseases. The imbalance between energy intake and expenditure as occur in obesity leads to mitochondrial dysfunction, characterized by a reduced ratio of energy production (ATP production) to respiration and increase of superoxide levels due excess electrons provided to mitochondrial respiratory chains and less ATP demand [5,6]. However, the increase of superoxide levels that potentially occur in obesogenic states did not trigger an increase of SOD enzyme levels. 
At contrary, epidemiological investigations have described association among metabolic dysfunctions as higher levels of body mass index (BMI), systolic and diastolic blood pressure, triglyceride, glucose, proinflammatory biomarkers and lower SOD activity [7-9].

Obesity has been related to several cardiovascular and metabolic disorders such as hypertension, type 2 diabetes, hyperinsulinemia, dyslipidemia, atherosclerosis, and even certain types of cancers. The obesity is related to energetic unbalance that triggered weight increase associated to increase in triglycerides storage, adipocyte proliferation and, in the first moment angiogenesis induction. This condition stimulated the production and secretion of several cytokines and other molecules related to inflammation process. Therefore, the obesity causes a chronic low-grade inflammation process that is a perpetuating factor of oxidative stress [10] and antioxidant capacity impairment [11]. As consequence of inflammatory oxidative process existent in the obese state the homeostasis of several physiological functions are affected inducing insulin resistance, hypercoagulability and atherogenesis, hypertension, increased risk of cardiovascular and thromboembolic events [12].

These data indicate that obesity and other metabolic diseases modulate the superoxide metabolism increasing the level of these ROS and decreasing the SOD activity. However, if this effect is related to genetic or just environmental factors is not completely known.

To contribute to the clarification of the potential genetic impact on SOD activity and its relation with metabolic diseases previous investigations have been performed. These studies evaluated an association between a genetic polymorphism present in the MnSOD gene enzyme and metabolic diseases and dysfunctions. This polymorphism localized in exon 2 (rs 4880) alters the structure and efficiency of this enzyme due the substitution of valine to alanine in the 16 codon of the gene. The Ala16Val substitution causes a conformational change from a $\beta$-sheet to $\alpha$-helix, inducing a $30 \%-40 \%$ increase in mitochondrial MnSOD activity [6]. The MnSOD Val16Ala variation has been reported to increase the risk of oxidative stress-related pathological conditions associated to both homozygous genotypes (AA and VV) due a MnSOD imbalance. The $\mathrm{V}$ allele presents higher superoxide radical levels than the $\mathrm{A}$ allele because its lower efficiency to dismutate this molecule into $\mathrm{H}_{2} \mathrm{O}_{2}$ [13].

Previously, Montano et al. [14] described positive association between VV genotype and obesity and other studies also described association with hypercholes-terolemia [15], diabetes or diabetic cardiovascular diseases $[16,17]$ diabetic nephropathy [18] and diabetic retinopathy [19], elevate plasmatic oxided-LDL levels mainly in diabetic patients [20], elevate also lipid peroxidation, protein carbonylation [10,21] and proinflammatory-cyto- kines (IL-1 $\beta$, IL-6, TNF $\alpha$ and Ig $\gamma$ ) [22].

These results suggests that Ala16Val MnSOD polymorphism can contribute to metabolic alterations related to obesity as well as the obesity can contribute to increase of superoxide anion and, subsequently to affect lipid-oxidative-inflammatory biomarkers that triggered other metabolic dysfunctions. To evaluate this hypothesis we performed an additional protocol that analyzed the effect of interaction between Ala16Val MnSOD polymorphism and obesity on lipid, oxidative and inflammatory biomarkers of health adult subjects.

\section{METHODS}

\subsection{Subjects and Study Design}

The Research Ethics Committee approved the study protocol (No. 537/02), and informed consent was obtained from all individuals whose information was collected prospectively. The study enrolled 161 subjects selected from a previous cross-sectional investigation that included 1058 participants [15].

\subsection{Inclusion and Exclusion Criteria}

The study enrolled subjects with follow inclusion criteria: obese (body mass index, BMI > $30 \mathrm{~kg} / \mathrm{m}^{2}$ ) and nonobese (BMI $<25 \mathrm{~kg} / \mathrm{m}^{2}$ ), and by central obesity defined as a waist circumference (WC) $>102 \mathrm{~cm}$ for men and $>88$ $\mathrm{cm}$ for women [23]. Exclusion criteria: Data from subjects without previous diseases and dysfunctions as hypotireoidism, hypercholesterolemia, stroke and neoplasias that could to influence results were excluded of the analysis. The smoker or subjects undergoing hypolipemic treatment or taking anti-inflammatory or other drugs that could alter lipid, oxidative and inflammatory blood markers were also excluded of the study. We also excluded subjects with differences in the nutritional pattern evaluated in the Montano's study [11] since these variables could to present some influence on blood biomarkers analyzed here.

\subsection{Blood Collection and Biochemical Analyzes}

Blood samples were collected after 12 h overnight fasting by venous puncture into gray and red top Vacutainers ${ }^{\circledR}$ (BD Diagnostics, Plymouth, UK) tubes. Plasma was used to measure the levels of fasting glucose and serum total cholesterol, and triglyceride concentrations were measured using standard enzymatic methods by use of Ortho-Clinical Diagnostics ${ }^{\circledR}$ reagents on the fully automated analyzer (Vitros $950{ }^{\circledR}$ dry chemistry system; Johnson \& Johnson, Rochester, NY, USA). High-density lipoprotein cholesterol was measured in the supernatant plasma after the precipitation of apolipoprotein B-containing lipoproteins with dextran sulfate and magnesium 
chloride as previously described [24]. Low-density lipoprotein cholesterol was estimated with the Friedewald equation [25]. High-sensitivity $C$ reactive protein (hsCRP) was measured by nephelometry (Dade Behring, Newark, DE, EUA). Serum thiobarbituric acid reactive substances (TBARS) were measured according to the modified method of Jentzsch et al. [26]. The carbonylation of serum proteins was determined by the Levine method with modifications [27]. Oxidized LDL (OxLDL) was also determined by capture ELISA according to the manufacturer's instructions (Mercodia AB, Uppsala, Sweden), as described by Holvoet et al. [28]. OxLDL autoantibodies (anti-OxLDL) were determined using ELISA as described by $\mathrm{Wu}$ and Lefvert [29]. The vitamin $\mathrm{C}$ and total polyphenols were espectrophotometrically evaluated as described in Montagner et al. [21].

\subsection{Ala16Val MnSOD Genetic Analysis}

The Ala16Val MnSOD gene polymorphism was determined as described in Montano et al. [14]. Briefly, genomic DNA was isolated from peripheral blood leukocytes using a DNA Mini Kit Purification (Mo Bio). The polymorphism was detected by PCR-RFLP analysis. PCR amplifications were performed using amplification primers (Gibco Inc, Co.) for a 110-bp fragment of the human MnSOD gene were

5'-ACCAGCAGGCAGCTGGCGCCGG-3'(sense strand) and 5'-GCGTTGATGTGAGGTTCCAG-3' (antisense strand). The PCR product was digested with Hae III (15U; $37^{\circ} \mathrm{C}, 6 \mathrm{~h}$, Gibco. Inc., Co.). A mutation was introduced by a primer mismatch to create a restriction cut site for Hae III in the 16 codon, and the following genotypes were observed: Ala16Ala (23 and 85 bp), Ala16Val (23, 85 and 110 bp) and Va16Val (110 bp).

\subsection{Statistics Analysis}

Statistical analysis was performed using the SPSS/PC statistical package, version 12.0 (SPSS, Inc., Chicago, IL). The lipid and oxidative-inflammatory biomarkers were compared between obese and nonobese groups by Student $\mathrm{T}$ test. Further the sample was regrouped in six categories considering the obese and Ala16Val MnSOD genotypes status: AA-nonobese (AANO), AA-obese (AAO), VV-nonobese (VVNO), VV-obese (VVO), AVnonobese (AVNO) and AV-obese (AVO). The biomarkers were compared again among these groups by analysis of variance One-Way followed by Bonferroni post hoc test. Complementary statistical analysis comparing frequencies distribution among the groups was analyzed by chi-square test. We also performed a multivariate analysis (logistic regression Backward wald method) to observe the possible influence of sex and age. All p values were two-tailed, and $\mathrm{p}<0.05$ was considered statis- tically significant.

\section{RESULTS}

The sample characteristic baselines are presented in Table 1. The mean age and sex distribution was similar in both groups. The nonobese subjects Ala16Val MnSOD genotype frequencies were: $\mathrm{AA}=26$ (33.3\%), $\mathrm{VV}=14$ (17.9\%), $\mathrm{AV}=38(48.7 \%)$ and in obese subjects were: $\mathrm{AA}=25$ (30.1\%), $\mathrm{VV}=19$ (22.9) and AV = 39 (47.0).

The biochemical biomarkers investigated here were compared between nonobese and obese and the results are described in Table 2.

The obese group presented higher values of LDLcholesterol total, triglycerides, hs-PCR, OxLDL and anti-

Table 1. Characteristics baseline of obese and no-obese subjects.

\begin{tabular}{|c|c|c|c|}
\hline & Obese $n=78$ & No obese $n=83$ & $\mathrm{p}$ \\
\hline Age (years, mean \pm SD) & $52.0 \pm 11.6$ & $50.2 \pm 11.9$ & 0.133 \\
\hline \multicolumn{4}{|l|}{ Gender } \\
\hline Male, $\mathrm{n}$ & 32 & 37 & 0.363 \\
\hline Female, $\mathrm{n}$ & 46 & 46 & \\
\hline BMI $\left(\mathrm{kg} / \mathrm{m}^{2}\right.$, mean $\left.\pm \mathrm{SD}\right)$ & $32.7 \pm 2.3$ & $22.7 \pm 3.1$ & 0.00001 \\
\hline $\begin{array}{l}\text { Waist circumference } \\
(\mathrm{cm}, \text { mean } \pm \text { SD) } \\
\text { Hypertension, } \mathrm{n}(\%)\end{array}$ & $101.1 \pm 8.9$ & $87.5 \pm 9.8$ & 0.00001 \\
\hline
\end{tabular}

Table 2. Comparison of biochemical biomarkers between obese and no obese subjects.

\begin{tabular}{|c|c|c|c|}
\hline Variables & & Mean \pm SD & $\mathrm{p}$ \\
\hline \multirow{2}{*}{ Cholesterol total (mg/dL) } & Nonobese & $202.4 \pm 62.9$ & 0.872 \\
\hline & Obese & $203.9 \pm 60.7$ & \\
\hline \multirow{2}{*}{ HDL-cholesterol (mg/dL) } & Nonobese & $48.8 \pm 11.3$ & 0.372 \\
\hline & Obese & $47.1 \pm 12.9$ & \\
\hline \multirow{2}{*}{ LDL-cholesterol (mg/dL) } & Nonobese & $106.7 \pm 51.4$ & 0.05 \\
\hline & Obese & $119.5 \pm 54.1$ & \\
\hline \multirow{2}{*}{ Triglycerides (mg/dL) } & Nonobese & $119.9 \pm 62.2$ & 0.003 \\
\hline & Obese & $153.9 \pm 74.7$ & \\
\hline \multirow{2}{*}{ Glucose (mg/dL) } & Nonobese & $89.9 \pm 43.3$ & 0.08 \\
\hline & Obese & $102.9 \pm 42.9$ & \\
\hline \multirow{2}{*}{ Hs-CRP (mg/L) } & Nonobese & $0.66 \pm 1.07$ & 0.001 \\
\hline & Obese & $1.51 \pm 1.89$ & \\
\hline \multirow{2}{*}{ OxLDL (mg/dL) } & Nonobese & $0.41 \pm 0.40$ & 0.003 \\
\hline & Obese & $0.63 \pm 0.52$ & \\
\hline \multirow{2}{*}{ anti-OxLDL (mg/L) } & Nonobese & $13.56 \pm 15.89$ & 0.007 \\
\hline & Obese & $20.77 \pm 17.29$ & \\
\hline \multirow[t]{2}{*}{ TBARS } & Nonobese & $6.10 \pm 4.27$ & 0.875 \\
\hline & Obese & $5.99 \pm 3.34$ & \\
\hline \multirow[t]{2}{*}{ Protein carbonylation } & Nonobese & $1.72 \pm 0.86$ & 0.391 \\
\hline & Obese & $1.51 \pm 0.39$ & \\
\hline \multirow[t]{2}{*}{ Thiols } & Nonobese & $154.0 \pm 147.6$ & 0.617 \\
\hline & Obese & $171.5 \pm 156.4$ & \\
\hline \multirow[t]{2}{*}{ Vitamin C } & Nonobese & $30.6 \pm 20.4$ & 0.955 \\
\hline & Obese & $30.8 \pm 18.5$ & \\
\hline \multirow[t]{2}{*}{ Total polyphenols } & Nonobese & $4.29 \pm 0.98$ & 0.09 \\
\hline & Obese & $4.69 \pm 1.14$ & \\
\hline
\end{tabular}

SD = standard deviation 
OxLDL than nonobese subjects. Multivariate analysis showed that differences of these variables between obese and no obese subjects were independent of sex and age.

The analysis of interaction between obesity with Ala16Val MnSOD polymorphism was performed grouping the sample in six categories that presented the follow distribution: AANO = 26 (16.1\%), VVNO = 14 (8.7\%), AVNO = 38 (23.6\%), AAO = 25 (15.5\%), VVO = 19 (11.8\%) and AVO = 39 (24.2\%).

The comparison of biochemical biomarkers among these six groups was also performed. The HDL-cholesterol, glucose, TBARS, protein carbonylation, thiols group, vitamin $\mathrm{C}$ and polyphenol totals were also similar among the groups. As we observed in the first analysis obese and nonobese group did not show influence of Ala16Val MnSOD genotypes.

However, the groups presented differences on cholesterol, triglycerides, LDL-cholesterol, hs-PCR, OxLDL and anti-OxLDL values. Figure 1 shows that cholesterol total and triglycerides were lower in AANO and AAO groups when compared to VVO group whereas the other groups showed intermediary values. The VVO and AVO presented higher levels of LDL-cholesterol when compared to obese and nonobese AA carrier's subjects. The other groups also presented intermediary values. The hsPCR and OxLDL levels were also higher in VVO and AVO subjects when compared with other groups including AAO subjects. The analysis of anti-OxLDL showed lower levels just to AANO subjects when compared to other groups. The VVNO, AVNO and AAO presented intermediary values but lower than VVO and AVO that also showed higher values of these biomarker.

Since the range of hs-CRP values was large, as can see by $\mathrm{SD}$ in Figure 1, we categorized these variables in lower and higher values ( $\geq 1 \mathrm{mg} / \mathrm{L})$ and compared among the six groups. The frequency of higher hs-CRP was $11.5 \%$ in AANO and $16.7 \%$ in AAO and these differences were not significant $(p=0.602)$. However, the obeses carriers VV and AV genotypes (VVO and AVO) presented significant number of subjects with higher hs-CPR than nonobeses. Similar analysis was performed by OxLDL biomarker comparing subjects with lower and higher value $(\geq 0.5 \mathrm{mg} / \mathrm{L})$ of this variable. The AANO presented lower frequency (23.1\%) of subjects with higher OxLDL values than other subjects (VVNO = $42.9 \%$; $\mathrm{AVNO}=52.6 \%, \mathrm{AAO}=40 \%$, $\mathrm{VVO}=52.6 \%$ and $\mathrm{AVNO}=61.8 \%, \mathrm{p}=0.006)$.
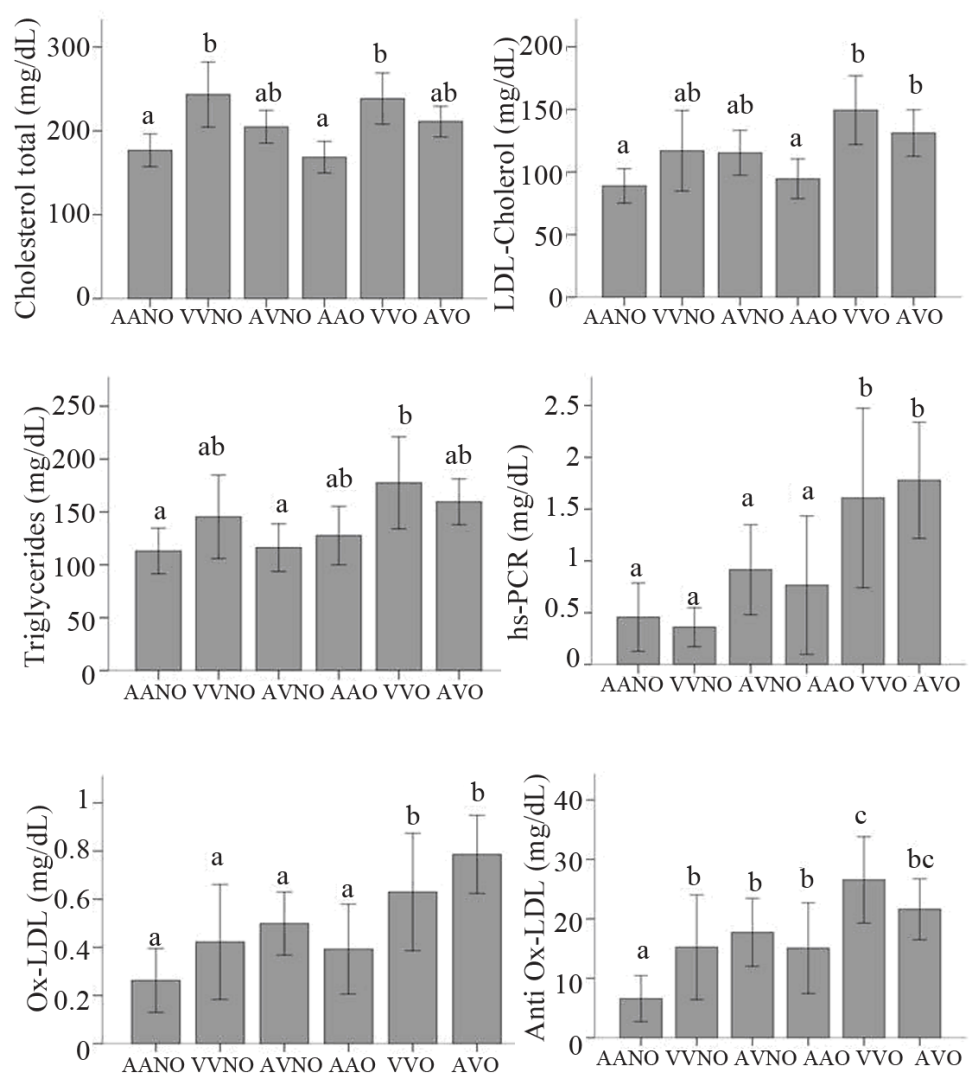

Figure 1. Comparison of cholesterol total, LDL-cholesterol and triglycerides, hs-PCR, OxLDL, anti-OxLDL among groups with different status of obesity and Ala16Val MnSOD genotypes. AANO = non obese AA subjects; VVNO = non obese VV subjects/AVNO = non obese VV subjects; AAO= obese AA; VVO= VV obese; AVO = AV obese. Different letters indicated significant statistical differences $(p<0.05)$. 


\section{DISCUSSION}

Our study evaluated whether the interaction between obesity and Ala16Val MnSOD polymorphism has some influence on oxidative and inflammatory biomarkers. To perform this analysis we selected a sample with similar health and lifestyle profile to minimize the influence of these variables on biomarkers investigated here.

This selection probably is reflected in the results of some variables related to oxidative metabolism that are directly influenced by environmental variables as dietary and pollutants factors such as the case of TBARS, protein carbonylation, thiols groups, vitamin $\mathrm{C}$ and total polyphenols that we found similar values among the groups.

In the present study, the comparison between obese and nonobese volunteers showed the occurrence of important differences in lipid, inflammatory and oxidative biomarkers since cholesterol total, LDL-cholesterol, triglycerides, hs-PCR, OxLDL and anti-OxLDL [30].

Currently is accepted that initiation, propagation, and development of obesity involves a chronic low-grade inflammation and oxidative stress processes [30-35]. This association occurs because the obesity is related to the storage of energy excess in adipocytes, resulting in both hypertrophy and hyperplasia which causes particularly mitochondrial stress. In this sense, oxidative stress can also be induced by adipocyte associated inflammatory macrophages that are reflected in the blood biomarkers differences observed in this study.

These results are not new since a large body of previous evidences has described the influence of obesity on lipid, oxidative and inflammatory biomarkers [30,36,37]. However, in our study we found that the alterations in these biomarkers can be influenced by the genetic status of volunteers considering the Ala16Val MnSOD polymorphism. Obese subjects V allele carrier's, in general presented more chance to have high values of cholesterol, LDL-cholesterol, triglycerides, hs-CRP, Ox-LDL and anti-OxLDL whereas AA carrier's presented a protective effect.

These results corroborated the previous study performed by Duarte et al. [15] that described association between the Ala16Val MnSOD polymorphism with hypercholesterolemia. Other studies as performed by Augusti et al. [38] also have described the association between imbalance in SOD activity in hypercholesterolemic subjects. We also found that triglycerides levels were higher in VVO, lower in AANO subjects whereas the other groups presented intermediary values between two groups. Previous investigation described the potential association between the MnSOD polymorphism and triglycerides levels as the study performed in Chinese patients with type 2 diabetes or diabetic CVD [16].
The biological effects of oxidized LDL (OxLDL) may contribute to initiation and progression of the atherosclerotic process, and the association between cardiovascular disease and oxidation of LDL has been largely demonstrated. A positive correlation of OxLDL concentration with cholesterol levels and body mass index, has been reported in previous studies $[30,33,37]$ indicating that metabolic syndrome and their components as obesity exacerbates oxidized LDL levels in a feedback loop [39]. The results found in the corroborates the relevance of oxidative stress status on OxLDL levels. However, these results suggest that oxidative stress associated to OxLDL levels can be also triggered by genetic factors since the OxLDL values were lower in AA obese and higher in VV obese subjects. This result confirms previous study performed by Gottlieb et al. [20] that found association between $\mathrm{V}$ allele with higher OxLDL levels mainly in diabetic elderlies.

Perhaps, the most important result showed here was related with the influence of the obesity and Ala16Val MnSOD polymorphism interaction on hs-CRP levels. Elevation of hs-CRP levels has been previously associated to obesity [40]. The concentration of this important inflammatory molecule was lower in nonobese carriers AA and VV genotypes when compared to VV and AV obese subjects. These results suggest that superoxide imbalance associated to V allele (VV or AV) could to be related to inflammatory plasmatic biomarkers. The result corroborates the in vitro study performed by Montano et al. [22] that evaluated cytokines production by human peripheral blood mononuclear cells (PBMCs) carrier's different Ala16Val-SOD2 genotypes and found higher levels of proinflammatory cytokines in VV-PBMCs when compared to AA-PBMCs.

Duarte et al. [15] also analyzed the potential effect of Ala16Val MnSOD polymorphism on hs-CRP levels. However, these authors did not find influence of the polymorphism in this variable since all hypercholesterolemic subjects presented higher hs-CRP levels when compared to control group independent of the Ala16Val MnSOD genotypes.

Evidences show that hs-CRP found in the blood rise in response to inflammation processes synthesized by the liver in response to factors released by macro-phages and fat cells (adipocytes) [41]. Additional investigations, as performed by Liu et al. [09] have suggested that hs-CRP may contribute to risk of cardiovascular disease by increasing oxidative stress.

The results described in this study corroborate the assumption that chronic low-grade inflammation probably occurs due changes in adipokines, secretion, fatty acidinduced inflammation, and also in the increase in oxidative stress [4]. This contribution potentially is triggered by activated macrophages which generate a range of 
ROS, including hydrogen peroxide, hypochlorous acid as well as superoxide [42]. Since superoxide is present in all cell types that participate in the inflammation process (leukocytes, endothelial, other vascular cells, etc.) if this ROS is not controlled may leads to cellular toxic effects. The chronic state of unbalance, as occurs in VV carriers, the superoxide could to produce important oxidative reactions contributing to oxidative-inflammatory process observed in the obesity. For example, the superoxide has a high affinity to nitric oxide (NO), a ROS that is produced by virtually all cells and is associated with several biological effects including the regulation of immune responses. When the superoxide anion reacts to molecules of nitric oxide, peroxynitrite is produced. Peroxynitrite formed by the diffusion rate-limited combination of nitric oxide (NO) and superoxide free radicals has been proposed to be a key contributor to oxidative damage, mainly because its highly reactive decomposition produces nitrogen dioxide, hydroxyl radical and carbonate radical [43]. These PN-derived radicals can oxidize proteins, nitrate tyrosine residues and induce cell membrane lipid peroxidation [44].

In conclusion, our data suggest that oxidative metabolism influenced by genetic status could minimize or maximize the obesity effects on lipid, oxidative and inflammatory biomarkers that are also implicated in the genesis of important dysfunctions and diseases as atherosclerosis, diabetes 2 and cardiovascular morbidities.

\section{REFERENCES}

[1] Valko, M., Rhodes, C.J., Moncol, J., Izakovic, M. and Mazur, M. (2006) Free radicals, metals and antioxidants in oxidative stress induced cancer. Chemico-Biological Interactions, 160, 1-4. doi:10.1016/j.cbi.2005.12.009

[2] Roberts, C.K. and Sindhu, K.K. (2009) Oxidative stress and metabolic syndrome. Life Sciences, 84, 705-712. doi:10.1016/j.lfs.2009.02.026

[3] Fridovich, I. (1986) Biologic effects of superoxide radical. Archives of Biochemistry and Biophisics, 247, 1-11. doi:10.1016/0003-9861(86)90526-6

[4] Christianson, D.W. (1997) Structural chemistry and biology of manganes metalloenzymes. Progress in Biophysics and Moecular Biology, 67, 217-252.

[5] Lenaz, G. (2001) The mitochondrial production of reactive oxygen species: Mechanisms and implications in human pathology. IUBMB Life, 52, 159-164. doi:10.1080/15216540152845957

[6] Kim, J.A., Wei, Y. and Sowers, J.R. (2008) Role of mitochondrial dysfunction in insulin resistance. Circulation Research, 102, 401-414.

[7] Isogawa, A., Yamakado, M., Masao Yano, M. and Shiba, T. (2009) Serum superoxide dismutase activity correlates with the components of metabolic syndrome or carotid artery intima-media thickness. Diabetes Research and Clinical Practice, 86, 213-218.

doi:10.1016/j.diabres.2009.09.007

[8] Karaouzene, N., Merzouk, H., Aribi, M., Merzouk, S.A., Berrouiguet, A.Y., Tessier, C. and Narce, M. (2011) Effects of the association of aging and obesity on lipids, lipoproteins and oxidative stress biomarkers: a comparison of older with young men. Nutrition Metabolic Cardiovascular Disease, 21, 792-799.

doi:10.1016/j.numecd.2010.02.007

[9] Liu, H., Yang, Y., Huang, G., Tan, S. and Liu, Y. (2012) Positive association of pro-inflammatory biomarkers and increased oxidative stress in the healthy elderly. Archives of Gerontology and Geriatrics, 54, e8-e12. doi:10.1016/j.archger.2011.05.016

[10] Higdon, J.V. and Frei, B. (2003) Obesity and oxidative stress: A direct link to CVD? Arteriosclerosis, Thrombosis, and Vascular Biology, 23, 365-367. doi:10.1161/01.ATV.0000063608.43095.E2

[11] Furukawa, S., Fujita, T., Shimabukuro, M., Iwaki, M., Yamada, Y., Nakajima, Y., Nakayama, O., Makishima, M., Matsuda, M. and Shimomura, I. (2004) Increased oxidative stress in obesity and its impact on metabolic syndrome. Journal of Clinical Investigation, 114, 17521761. doi:10.1172/JCI21625

[12] Hauner, H. (2004) The new concept of adipose tissue function. Physiology and Behavior, 83, 653-658. doi:10.1016/j.physbeh.2004.09.016

[13] Sutton, A., Khoury, H., Prip-Buus, C., Cepanec, C., Pessayre, D. and Degoul, F. (2003) The Ala16Val genetic dimorphism modulates the import of human manganese superoxide dismutase into rat liver mitochondria. Pharmacogenetics, 13, 145-157. doi:10.1097/00008571-200303000-00004

[14] Montano, M.A.E., Lera, J.P.B., Gottlieb, M.G.V., Schawnke, C.H.A., Rocha, M.I.U.M., Manica-Cattani, M.F., Santos, G. F. and Cruz, I.B.M. (2009) Association between manganese superoxide dismutase (MnSOD) gene polymorphism and elderly obesity. Molecular Cellular Biochemistry. 328, 33-40. doi:10.1007/s11010-009-0071-z

[15] Duarte, M.M.F., Duarte, M.M.F., Moresco, R.N., Duarte, T., Santi, A., Bagatini, M.D., Da Cruz, I.B.M., Schetinger, M.R.C. and Loro, V.L. (2010) Oxidative stress in hypercholesterolemia and its association with Ala16Val superoxide dismutase gene polymorphism. Clinical Biochemistry, 43, 1118-1123. doi:10.1016/j.clinbiochem.2010.07.002

[16] Chen, H., Yu, M., Li, M., Zhao, R., Zhu, Q., Zhou, W., et al. (2012) Polymorphic variations in manganese superoxide dismutase (MnSOD), glutathione peroxidase-1 (GPX1), and catalase (CAT) contribute to elevated plasma triglyceride levels in Chinese patients with type 2 diabetes or diabetic cardiovascular disease. Molecular Cellular Biochemistry. 363, 85-91. doi:10.1007/s11010-011-1160-3

[17] Jones, D.A., Prior, S.L., Tang, T.S., Bain, S.C., Hurel, S.J., Humphries, S.E., et al. (2010) Association between the rs4880 superoxide dismutase $2(\mathrm{C}>\mathrm{T})$ gene variant and coronary heart disease in diabetes mellitus. Diabetes $R e$ - 
search and Clinical Practice, 90, 196-201. doi:10.1016/j.diabres.2010.07.009

[18] Mollsten, A., Marklund, S.L., Wessman, M., Svensson, M., Forsblom, C., Parkkonen, M., et al. (2007) A functional polymorphism in the manganese superoxide dismutase gene and diabetic nephropathy. Diabetes, 56, 265269. doi:10.2337/db06-0698

[19] Kangas-Kontio, T., Vavuli, S., Kakko, S.J., Penna, J., Savolainen, E.R., Savolainen, M.J. and Liinamaa, M.J. (2009) Polymorphism of the manganese superoxide dismutase gene but not of vascular endothelial growth factor gene is a risk factor for diabetic retinopathy. British Journal of Ophthalmology, 93, 1401-1416.

doi:10.1136/bjo.2009.159012

[20] Gottlieb, M.G., Schwanke, C.H., Santos, A.F., Jobim, P.F., Denise P. Müssel, D.P., Ivana B.M. and da Cruz, I.B.M. (2005) Association among oxidized LDL levels, SOD2, apolipoprotein E polymorphisms and cardiovascular risk factors in a south Brazilian region population. Genetic Molecular Research, 4, 691-703.

[21] Montagner, G.F.F., Sagrillo, M., Machado, M.M., Almeida, R.C., Mostardeiro, C.P., Duarte, M.F.F. and Cruz, I.B.M. (2010) Toxicological effects of ultraviolet radiation on lymphocyte cells with different manganese superoxide dismutase Ala16Val polymorphism genotypes. Toxicology in Vitro, 24, 1410-1416. doi:10.1016/j.tiv.2010.04.010

[22] Montano, M.A.E., Cruz, I.B.M., Duarte, M.M.M.F., Krewer, C.C., Rocha, M.I.U.M., Manica-Cattani, M.F., Soares, F.A.A., Rosa, G., Maris, A.F., Battiston, F.G., Trott, A. and Lera, J.P.B. (2012) Inflammatory cytokines in vitro production are associated with A-la16Val superoxide dismutase gene polymorphism of peripheral blood mononuclear cells. Cytokine, 60, 30-33. doi:10.1016/j.cyto.2012.05.022

[23] World Health Organization (1988) Obesity: Preventing and managing the global epidemic: Report of a WHO consultation on obesity. WHO Publications, Geneva.

[24] Bachorik, P.S. and Albers, J.J. (1986) Precipitation methods for quantification of lipoproteins. Methods in Enzymology, 129, 78-100. doi:10.1016/0076-6879(86)29063-1

[25] Friedewald, W.T., Levy, R.I. and Fredrickson, D.S. (1972) Estimation of the concentration of low density lipoprotein cholesterol in plasma, without the use of preparative ultracentrifuge. Clinical Chemistry, 18, 499-502.

[26] Jentzsch, A.M., Bachmann, H., Fürst, P. and Biesalski, H.K. (1996) Improved analysis of malondialdehyde in human body fluids. Free Radical Biology and Medicine, 20, 251-256. doi:10.1016/0891-5849(95)02043-8

[27] Levini, R.L., Garland, D., Oliver, C.N., Amiei, A., Climent, I. and Lenz, A. (1990) Determination of car-bonyl content in oxidatively modified proteins. Methods in Enzymology, 186, 464-468. doi:10.1016/0076-6879(90)86141-H

[28] Holvoet, P., Stassen, J.M., Van Cleemput, J., Collen, D. and Vanhaecke, J. (1998) Oxidized low density lipoproteins in patients with transplantassociated coronary artery disease. Arterioscleroses, Thrombosis and Vascular Bi- ology, 18, 100-107. doi:10.1161/01.ATV.18.1.100

[29] Wu, R. and Lefvert, A.K. (1995) Autoantibodies against oxidized low density lipoprotein (OxLDL): Characterization of antibody isotype, subclass, affinity and effect on the macrophage uptake of oxLDL. Clinical and Experimental Immunology, 102, 174-180. doi:10.1111/j.1365-2249.1995.tb06652.x

[30] Holvoet, P., Kritchevsky, S.B., Tracy, R.P., Mertens, A., Rubin, S.M., Butler, J., Goodpaster, B. and Harris, T.B. (2004) The metabolic syndrome, circulating oxidized LDL, and risk of myocardial infarction in well-functioning elderly people in the Health, Aging, and Body Composition cohort. Diabetes, 53, 1068-1073. doi:10.2337/diabetes.53.4.1068

[31] Fonseca, V., Desouza, C., Asnani, S. and Jialal, I. (2004) Nontraditional risk factors for cardiovascular disease in diabetes. Endocrine Reveiws, 25, 153-175. doi:10.1210/er.2002-0034

[32] Martí, A., Marcos, A. and Martínez, J.A. (2001) Obesity and immune function relationships. Obesity Reveiws, 2, 131-140. doi:10.1046/j.1467-789x.2001.00025.x

[33] Hotamisligil, G.S. (2006) Inflammation and metabolic disorders. Nature, 444, 860-867. doi:10.1038/nature05485

[34] Conen, D., Rexrode, K.M., Creager, M.A., Ridker, P.M. and Pradhan, A.D. (2009) Metabolic syndrome, inflammation, and risk of symptomatic peripheral artery disease in women: A prospective study. Circulation, 120, 10411047. doi:10.1161/CIRCULATIONAHA.109.863092

[35] De Heredia, F.P., Gómez-Martínez, S. and Marcos, A. (2012) Obesity, inflammation and the immune system. Proceeding of the Nutrition Society, 71 332-338. doi:10.1017/S0029665112000092

[36] Ozata, M., Mergen, M., Oktenli, C., Aydin, A., Sanisoglu, S.Y., Bolu, E., Yilmaz, M.I., Sayal, A., Isimer, A. and Ozdemir, I.C. (2002) Increased oxidative stress and hypozincemia in male obesity. Clinical Biochemistry, 35, 627-631. doi:10.1016/S0009-9120(02)00363-6

[37] Burgos Alves, M.I., Avilés Plaza, F., Martínez-Tomás, R., Sánchez-Campillo, M., Larqué, E., Pérez-Llamas, F., Martínez Hernández, P., Parra Pallarés, S. (2010) Oxidized LDL and its correlation with lipid profile and oxidative stress biomarkers in young healthy Spanish subjects. Journal of Physiology Biochemistry, 66, 221-227. doi:10.1007/s13105-010-0028-4

[38] Augusti, P.R, Ruviaro, A.R., Quatrin, A., Somacal, S., Conterato, G.M., Vicentini, J.T., Duarte, M.M. and Emanuelli, T. (2012) Imbalance in superoxide dismutase/thioredoxin reductase activities in hypercholesterolemic subjects: Relationship with low density lipoprotein oxidation. Lipids in Health and Diseases, 11, 79. doi:10.1186/1476-511X-11-79

[39] Holvoet, P., De Keyzer, D. and Jacobs Jr., D.R. (2008) Oxidized LDL and the metabolic syndrome. Future Lipidology, 3, 637-649. doi:10.2217/17460875.3.6.637

[40] Phillips, C.M., Tierney, A.C., Perez-Martinez, P., Defoort, C., Blaak, E.E., Gjelstad, I.M., Lopez-Miranda, J., KiecKlimczak, M., Malczewska-Malec, M., Drevon, C.A., Hall, W., Lovegrove, J.A., Karlstrom, B., Risérus, U. and 
Roche, H.M. (2012) Obesity and body fat classification in the metabolic syndrome: Impact on cardiometabolic risk metabotype. Obesity. doi:10.1038/oby.2012.188

[41] Bahceci, M., Gokalp, D., Bahceci, S., Tuzcu, A., Atmaca, S. and Arikan, S. (2007) The correlation between adiposity and adiponectin, tumor necrosis factor alpha, interleukin-6 and high sensitivity C-reactive protein levels. Is adipocyte size associated with inflammation in adults? Journal of Endocrinological Investigation, 30, 210-214.

[42] Ferrari, C.K., Souto, P.C., França, E.L. and HonorioFrança, A.C. (2011) Oxidative and nitrosative stress on phagocytes' function: From effective defense to immunity evasion mechanisms. Archivum Immunologiae et Therapia Experimentalis, 59, 441-448. doi:10.1007/s00005-011-0144-z

[43] Alvarez, M.N., Trujillo, M. and Radi, R. (2002) Peroxynitrite formation from biochemical and cellular fluxes of nitric oxide and superoxide. Methods in Enzymology, 359, 353-366. doi:10.1016/S0076-6879(02)59198-9

[44] Denicola, A. and Radi, R. (2005) Peroxynitrite and drugdependent toxicity. Toxicology, 208, 273-288. doi:10.1016/j.tox.2004.11.023 